\title{
VARIABILIDAD TEMPORAL DE ALGUNAS PROPIEDADES QUÍMICAS EN UN SUELO SOMETIDO A DISTINTAS SUCESIONES DE CULTIVO ${ }^{1}$
}

\author{
OROZIMBO SILVEIRA CARVALHO², ANTONIO SAA ${ }^{3}$, JOSE MARIA GASCÓ MONTES ${ }^{3}$, \\ RAFAEL CABALLERO ${ }^{4}$ y FRANCISCA GUERRERO LÓPEZ ${ }^{5}$
}

\begin{abstract}
RESUMEN - El presente trabajo ha sido realizado en la Finca Experimental "La Poveda" en Arganda del Rey (Madrid), con las siguientes coordenadas geográficas: latitud 40¹9' N; longitud $3^{\circ} 19^{\prime} \mathrm{W}$ Gr; y altitud $550 \mathrm{~m}$. El objetivo principal consiste en el estudio de la variabilidad temporal de algunas propiedades químicas en un suelo sometido a distintas sucesiones de cultivo. Para el estudio de la variabilidad temporal de las características químicas el muestreo se realizó en los meses de febrero y septiembre de 1993 y febrero y septiembre de 1994. La materia orgánica del sistema Prado/Veza-Avena presenta el menor coeficiente de variación con respecto a los sistemas monocultivo de Cebada y Veza-Avena/Girasol. El menor contenido de materia orgánica a lo largo del tiempo lo presenta el sistema Cebada/Cebada. Los sistemas Prado/Veza-Avena y Veza-Avena/Girasol presentaron contenidos de materia orgánica 42,86\% y 40,54\% superiores al del sistema Cebada/Cebada.
\end{abstract}

Términos para indexación: materia orgánica, ácidos húmicos, sistemas agrícolas.

\section{TEMPORAL VARIABILITY OF CHEMICAL PROPERTIES IN A SOIL WITH THREE CULTIVATION SYSTEMS}

\begin{abstract}
This study has been carried out at the Experimental Station "La Poveda" in Arganda del Rey (Madrid), with the following geographical coordinates: latitude $40^{\circ} 19^{\prime} \mathrm{N}$; longitude $3^{\circ} 19^{\prime} \mathrm{W}$ Gr; and altitude $550 \mathrm{~m}$. The main objective was to study the temporal variability of chemical properties in a soil with three cultivation systems. In order to study variation with time of chemical characteristics, samples were taken in February and September 1993 and February and September 1994. Organic matter content in the prairie/vetch-oat system had the smallest variation coefficient with respect to barley and vetch-oat/sunflower systems. Along with the time the barley/barley system presented a lower organic matter content. The crop systems pasture/vetch-oat and vetch-oat/sunflower presented an organic matter content 42.86 and $40.54 \%$ higher than that presented by barley/barley system, respectively.
\end{abstract}

Index terms: organic matter, humic acid, agricultural systems.

\footnotetext{
${ }^{1}$ Aceptado para publicación en 18 de noviembre de 1998. Extraída de la tesis del primero autor presentada en la Univerdidad Politécnica de Madrid.

${ }^{2}$ Ing. Agr., Dr., Embrapa-Centro Nacional de Pesquisa de Algodão (CNPA), Caixa Postal 174, CEP 58107-720 Campina Grande, PB. E-mail: orozimbo@cnpa.embrapa.br

${ }^{3}$ Ing. Agr., Dr., Dep. de Edafologia, Escuela Técnica Superior de Ingenieros Agrónomos de la Universidad Politécnica de Madrid, Avenida Complutense S/N, 28040, Madrid.

${ }^{4}$ Ing. Agr., Dr., Finca Experimental La Poveda, Consejo Superior de Investigaciones Científicas. 28500, Arganda del Rey, Madrid.

${ }^{5}$ Quím., Drª ${ }^{\mathrm{a}}$, Dep. de Edafologia, Escuela Técnica Superior de Ingenieros Agrónomos de la Universidad Politécnica de Madrid. E-mail: fguer@eda.etsia.upm.es
}

\section{INTRODUCCIÓN}

La importancia de la materia orgánica en el mantenimiento de las propiedades químicas adecuadas para el buen desarrollo de los cultivos es un hecho incuestionable, además de que su conocimiento detallado en un área de cultivo es fundamental para la toma de decisiones en un proceso productivo en equilibrio con el medio ambiente.

Aunque la materia orgánica del suelo está formada por carbono, hidrógeno, oxígeno, nitrógeno, azufre y fósforo, la mayor parte de los estudios realizados se refieren a los contenidos de carbono y 
nitrógeno y, con menor frecuencia, a los de azufre y fósforo, debido a las dificultades analíticas que presenta la determinación del oxígeno e hidrógeno orgánicos del suelo (Wild, 1992).

El estudio de la dinámica de la materia orgánica es de particular interés en las áreas semiáridas situadas en las zonas templadas. En España, según Urbano \& Moro (1992), y con base a los datos del Anuario de Estadística Agraria (1990), existen $48 \times 10^{6}$ ha de superficie agrícola total, de los cuales $20,3 \times 10^{6}$ ha están ocupadas por sistemas agrícolas con laboreo habitual, incluyendo cultivos herbáceos (11,3 x $10^{6}$ ha) y arbóreos ( 4,7 x $10^{6}$ ha) más los barbechos (4,3 x $10^{6}$ ha), que equivalen al $43,5 \%$ de la superficie agrícola útil (SAU), además de los sistemas pratícolas y pascícolas, representados por las praderas naturales y artificiales $\left(1,4 \times 10^{6}\right.$ ha) y los pastizales $\left(5,3 \times 10^{6}\right.$ ha) que se extienden sobre un total de $6,7 \times 10^{6}$ ha que representa el $14,6 \%$ de la SAU.

Si consideramos en conjunto los sistemas con laboreo habitual y los sistemas pratícolas y pascícolas, podemos verificar que ocupan un área de $27 \times 10^{6}$ ha, lo que representa $58,1 \%$ de la SAU, lo que, por si solo da una idea de la importancia de estos dos sistemas agrícolas en la economía agrícola española.

En estos sistemas agrícolas normalmente hay un empobrecimiento gradual en materia orgánica como consecuencia del desarrollo de los cultivos intensivos, en los cuales se utilizan cantidades crecientes de fertilizantes para mantener los niveles de productividad (Hernando, 1987).

Los suelos agrícolas, que se definen como aquellos capaces de producir elevados rendimientos de una variedad indefinida de cultivos bajo condiciones adecuadas de laboreo, presentan en España contenidos medios de materia orgánica del 1\% (Lobo, 1985), lo que hace patente la necesidad de definir alternativas de aporte de materia orgánica, para llevar el nivel a un 2\% (mínimo aceptable) o un $3 \%$ que sería lo deseable (Hernando, 1987).

De lo expuesto se puede percibir la importancia que pueden tener estudios dirigidos a un mejor entendimiento de la dinámica de la materia orgánica en los suelos de las zonas semiáridas templadas, incluyendo evaluaciones de su variabilidad temporal, en función del área ocupada por cultivos en estas regiones, en los distintos sistemas agrícolas.
La materia orgánica es de importancia para el suelo, no solo como fuente de nutrientes, sino también porque aumenta la capacidad de intercambio catiónico (Jorge, 1986).

Por lo tanto, para el desarrollo de una "agricultura sostenible" es importante la adopción de un manejo adecuado del suelo con el objetivo de estimular la actividad de los microorganismos y animales presentes en él, lo que puede realizarse a través del mantenimiento de un nivel aceptable de materia orgánica, que puede contribuir para que el suelo contenga mayores reservas de nutrientes para las plantas. Además ayuda a prevenir extremos de acidez o alcalinidad del suelo (FAO, 1991).

En este sentido, las prácticas culturales y de manejo, tales como rotación de cultivos, reciclado de abonos y laboreo de conservación, para controlar la erosión del suelo y las pérdidas de nutrientes y mantener o mejorar la productividad del suelo, son muy aceptables (López-Fando, 1991).

El tipo de suelo, el clima y el sistema de cultivo determinan el punto de equilibrio que alcanza el nivel de materia orgánica en el suelo. Si inicialmente, el nivel está por debajo o por encima de este punto de equilibrio hay un incremento o una pérdida hasta que se llega al nivel de equilibrio. Así por ejemplo, si se ara un prado permanente para un cultivo agrícola, habrá una pérdida gradual de materia orgánica, lo que puede continuar durante más de treinta años, hasta que se establezca el nuevo nivel ( Davies et al., 1987).

La materia orgánica en general y la fracción humificada en particular, están intimamente ligados a la fertilidad del suelo y al desarrollo vegetal mediante una serie de acciones de naturaleza física, químico-física, química y biológica que ejercen sobre el mismo suelo, así como otras de tipo fisiológico que efectúan sobre la planta (Costa et al., 1991).

Según Melo et al. (1994), la relación C-orgánico de los ácidos fúlvicos/C-orgánico de los ácidos húmicos es variable, ya que depende de la química del suelo y del período del año, con posibilidad de invertirse al cambiar de una estación de sequía a una de lluvias.

Durante los primeros estadios de la humificación, la naturaleza y propiedades de la materia orgánica del suelo vienen condicionadas principalmente por la propia composición química de la biomasa aportada por los vegetales (Almendros et al., 1982). 
La principal hipótesis de este trabajo es que las distintas sucesiones de cultivo producen cambios en la dinámica de la materia orgánica y en algunas propiedades químicas relacionadas directa o indirectamente con la fracción orgánica del suelo.

\section{MATERIAL Y MÉTODOS}

El presente trabajo ha sido realizado en la Finca Experimental "La Poveda" del Consejo Superior de Investigaciones Científicas (CSIC), en Arganda del Rey (Madrid). La finca experimental "La Poveda" se encuentra situada en el centro de España, al SE de la ciudad de Madrid con las siguientes coordenadas geográficas: latitud $40^{\circ} 19^{\prime} \mathrm{N}$; longitud $3^{\circ} 19^{\prime} \mathrm{W}$ Gr; y altitud $550 \mathrm{~m}$, con clima mesomediterráneo: temperatura media anual $=13,1^{\circ} \mathrm{C}$, precipitación media anual de 464,3 $\mathrm{mm}$ y evapotranspiración media anual según Thornthwaite de $737 \mathrm{~mm}$.

Las muestras fueron cogidas en tres parcelas distintas: 1) Una parcela con once años de rotación de los cultivos Cebada/Veza-Avena/Trigo (Sistema I), abonada con $400 \mathrm{~kg} / \mathrm{ha}$ de la fórmula 8-15-15 en fundación, más $50 \mathrm{~kg} / \mathrm{ha}$ de $\mathrm{N}$ en cobertera; 2) Una parcela con once años de rotación de los cultivos Maíz/Cebada/Veza-Cebada/ Veza-Avena/Girasol (Sistema II), abonada con $400 \mathrm{~kg} / \mathrm{ha}$ de la fórmula 8-15-15 en fundación, más $100 \mathrm{~kg} / \mathrm{ha}$ de $\mathrm{N}$ en cobertera; 3 ) Una pradera sometida a pastoreo durante diez años con una carga animal de 50 ovejas/ha, sembrada inicialmente con alfalfa, trébol blanco, festuca y dáctilo. A partir del año 10 la pradera ha sido sustituída por el cultivo Veza-Avena (Sistema III), abonada con $600 \mathrm{~kg} / \mathrm{ha}$ de la fórmula 8-15-15 en el inicio de la estación de crecimiento, más $100 \mathrm{~kg} / \mathrm{ha}$ de $\mathrm{N}$ antes de los cortes de abril y agosto de cada año.

Los cultivos de Veza-Avena, tanto en las parcelas del Sistema I como en las parcelas del Sistema II, no fueron abonados.

Para las determinaciones relativas a la variación temporal de las características químicas se han realizado muestreos en el horizonte superficial de $20 \mathrm{~cm}$ de espesor en los meses de febrero y septiembre de 1993 y febrero y septiembre de 1994. En cada sistema y época se han tomado cinco muestras (con cuatro submuestras por cada punto de muestreo) espaciadas $6 \mathrm{~m}$. La distancia de 6 m entre muestras se definió en función del análisis variográfico realizado en el primer muestreo (Carvalho, 1996). En el laboratorio las muestras fueron secadas al aire durante el tiempo necesario según el estado de humedad de la muestra. A continuación las muestras fueron tamizadas en un tamiz con una malla de $2 \mathrm{~mm}$. Todos los datos analíticos se refieren al peso seco determinado en estufa a $105^{\circ} \mathrm{C}$ hasta pesada constante, efectuándose las determinaciones analíticas por triplicado. La medida de la conductividad eléctrica fue hecha en el extracto acuoso $(1: 2,5)$ con un conductímetro CRISON 525. El pH se determinó en el mismo extracto acuoso del suelo empleado para medir la conductividad eléctrica, con un pHmetro CRISON 2000. El carbono total se ha determinado por calcinación directa a $540^{\circ} \mathrm{C}$ de acuerdo con la recomendación de los Métodos Oficiales de Análisis (Espanha, 1986). La materia orgánica oxidable se ha determinado por el método de oxidación por vía húmeda propuesto por los Métodos Oficiales de Análisis (Espanha, 1986). El carbono orgánico se obtiene dividiendo por 1,72. Para estudiar la evolución de la materia orgánica del suelo se ha procedido a la determinación del carbono del extracto húmico total (EHT), extraído según la técnica de Dabin (1971). La determinación del nitrógeno orgánico se lleva a cabo según el método Kjeldahl, mientras que, la capacidad de intercambio catiónico se ha determinado en una solución de acetato amónico $1 \mathrm{~mol} / \mathrm{L} \mathrm{pH} \mathrm{7,0.}$

El análisis estadístico de los datos obtenidos para las propiedades químicas en los tres sistemas de cultivo y en las cuatro épocas de muestreo fueron llevados a cabo por medio del programa estadístico SAS (SAS Institute, 1985). Los datos referentes a los parámetros químicos relativos al estudio de la variabilidad temporal fueron sometidos a análisis de varianza (ANOVA) y las medias de estos parámetros fueron comparadas por el test de Bonferroni al nivel de 5\%. También se realizó un estudio de correlación entre los parámetros.

\section{RESULTADOS Y DISCUSIÓN}

Los valores de conductividade eléctrica (Cuadro 1) fueron más elevados en la sucessión de cultivo Prado/Veza-Avena (Sistema III), seguidos de la sucessión Vez-Avena/Girasol (Sistema II) y los más bajos valores fueron obtenidos para el sistema de cultivo Cebada/Cebada (Sistema I). En relación al factor época de muestreo, los mayores valores referentes a la conductividad eléctrica fueron obtenidos en el verano de 1994, lo que puede estar relacionado com las precipitaciones pluviométricas en el período de los muestreos. Los valores de lluvia entre los años de 1975 y 1994 ponem de manifiesto que entre febrero de 1993 y septiembre de 1994 fue un período de gran seguía, con un total de lluvias en el año de 1993 del 77,81\% y en el año de 1994 ha llovido $53,50 \%$ de la media de los últimos 20 años $\left(456,1 \mathrm{~L}^{*} \mathrm{~m}^{-2}\right)$.

En relación a la mediana de los últimos 20 años $\left(463,65 \mathrm{~L}^{*} \mathrm{~m}^{-2}\right)$ los valores correspondientes a la lluvia en los años de 1993 y 1994 fueron, respectivamente, 
$76,54 \%$ y $52,62 \%$. Además, en el período comprendido entre febrero y septiembre de 1994 la lluvia correspondió a solamente el 35,14\% de la media del mismo período relativo a los años de 1975 y 1994.

En los períodos de sequía es normal un aumento en la conductividad eléctrica al aumentar la concentración de sales en la solución del suelo, como consecuencia de la disminución de la humedad debido a la evapotranspiración (Fuentes, 1992).

Los valores de $\mathrm{pH}$ (Cuadro 2) son más elevados en la sucessión Cebada/Cebada (Sistema I), concordante con los valores más bajos de la conductividad eléctrica.

Con relación a las épocas de muestreo también se observaron diferencias significativas principalmente entre las muestras de invierno y verano, debido a la absorción de nutrientes durante el período de cultivo, induciendo a una disminución del grado de saturación del complejo de cambio (Duchaufour, 1987 y Bernal et al., 1992). También puede ser atribuído al aumento de la respiración radicular.

El menor contenido de materia orgánica (Cuadro 3) lo encontramos en el sistema I (Cebada/Cebada), seguido del sistema II (Veza-Avena/Girasol). El de mayor contenido es el sistema III, Prado/Veza-Avena.

El contenido medio de materia orgánica (Cuadro 3) en los sistemas III y II fue $42,86 \%$ y $40,54 \%$ superior al valor detectado en el sistema I. A excepción del muestreo de septiembre/93, siempre se mantiene con mayor nivel de materia orgánica el sistema III, seguido del II y siempre, el de menor contenido, es el sistema I (Cebada/Cebada).
Los valores de carbono orgánico total (Cuadro 4) no siguen el comportamiento en cuanto a materia orgánica (Cuadro 3), ya que los sistemas II y III no presentan diferencias significativas, atribuible a la retirada del prado aproximadamente ocho meses antes del muestreo de febrero del 94, no notándose aún la influencia del cultivo de Veza-Avena, al no quedar restos vegetales, concordante con el bajo contenido de septiembre del mismo año $(5,73 \%)$.

En cuanto al fraccionamiento del carbono orgánico (Cuadros 5 y 6), el contenido de carbono del EHT es diferente entre sistemas, concordante con el porcentaje de materia orgánica (Cuadro 3), poniéndose en evidencia el distinto manejo del suelo. Entre muestreos se encuentran diferencias significativas en el contenido de carbono en los ácidos húmicos.

En el sistema I (Cebada/Cebada), es donde son menores los contenidos en materia orgánica, carbono orgánico total, extracto húmico total y ácidos húmicos, con diferencias significativas con relación a los sistemas II y III (Cuadros 3, 4, 5 y 6).

El contenido en nitrógeno (Cuadro 7) muestra su valor más bajo en el sistema I, tanto al final como durante todas las épocas de muestreo, así como el de la capacidad de intercambio catiónico (Cuadro 8), concordante con el menor valor de materia orgánica (González et al., 1977).

Las relaciones $\mathrm{C} / \mathrm{N}$ (Cuadro 9) se mantienen iguales entre sí en los distintos sistemas, evidenciándose el equilibrio alcanzado en la evolución de la materia orgánica del suelo.

Para evaluar el grado de humificación de la materia orgánica del suelo, se utiliza el índice $\mathrm{C}_{\mathrm{OX}} / \mathrm{C}_{\mathrm{EHT}}$

CUADRO 1. Valores medios de conductividad eléctrica $\left(\mu \mathrm{S} / \mathrm{cm}\right.$ a $\left.25^{\circ} \mathrm{C}\right)$ en los sistemas de cultivo Cebada/Cebada (Sistema I), Veza-Avena/Girasol (Sistema II) y Prado/Veza-Avena (Sistema III) en distintas épocas de muestreo. Madrid, 1996².

\begin{tabular}{clllll}
\hline \multirow{2}{*}{ Sistemas } & \multicolumn{5}{c}{ Conductividad eléctrica $\left(\mu \mathrm{S} / \mathrm{cm}\right.$ a $\left.25^{\circ} \mathrm{C}\right)$} \\
\cline { 2 - 5 } & Feb.-93 & Sep.-93 & Feb.-94 & Sep.-94 & Media \\
\hline I & 326,33 & 287,67 & 269,67 & 385,33 & $317,25 \mathrm{C}$ \\
II & 443,67 & 262,67 & 244,00 & 475,67 & $356,50 \mathrm{~B}$ \\
III & 423,00 & 528,00 & 383,67 & 553,67 & $472,08 \mathrm{~A}$ \\
Media & $397,67 \mathrm{~b}$ & $359,44 \mathrm{c}$ & $299,11 \mathrm{~d}$ & $471,56 \mathrm{a}$ & 381,94 \\
\hline
\end{tabular}

${ }^{1}$ Los valores seguidos por la misma letra minúscula en la fila y mayúsculas en la columna no son significativamente diferentes por el test de Bonferroni al nivel de 5\% de probabilidad. 
CUADRO 2. Valores medios de pH en los sistemas de cultivo Cebada/Cebada (Sistema I), Veza-Avena/Girasol (Sistema II) y Prado/Veza-Avena (Sistema III) en distintas épocas de muestreo. Madrid, 19961.

\begin{tabular}{cccccc}
\hline Sistemas & \multicolumn{5}{c}{$\mathrm{pH}$} \\
\cline { 2 - 5 } & Feb.-93 & Sep.-93 & Feb.-94 & Sep.-94 & Media \\
\hline I & 7,77 & 7,93 & 7,79 & 7,85 & $7,84 \mathrm{~A}$ \\
II & 7,58 & 7,89 & 7,73 & 7,79 & $7,75 \mathrm{~B}$ \\
III & 7,81 & 7,83 & 7,61 & 7,69 & $7,73 \mathrm{~B}$ \\
Media & $7,72 \mathrm{c}$ & $7,88 \mathrm{a}$ & $7,71 \mathrm{c}$ & $7,78 \mathrm{~b}$ & 7,77 \\
\hline
\end{tabular}

${ }^{1}$ Los valores seguidos por la misma letra minúscula en la fila y mayúsculas en la columna no son significativamente diferentes por el test de Bonferroni al nivel de $5 \%$ de probabilidad.

CUADRO 3. Valores medios del contenido de materia orgánica $(\mathrm{g} / \mathrm{kg})$ en los sistemas de cultivo Cebada/ Cebada (Sistema I); Veza-Avena/Girasol (Sistema II) y Prado/Veza-Avena (Sistema III) en distintas épocas de muestreo. Madrid, 1996'.

\begin{tabular}{cccccc}
\hline Sistemas & \multicolumn{5}{c}{ Materia orgánica $(\mathrm{g} / \mathrm{kg})$} \\
\cline { 2 - 5 } & Feb.-93 & Sep.-93 & Feb.-94 & Sep.-94 & Media \\
\hline I & 12,3 & 14,0 & 13,0 & 13,3 & $13,2 \mathrm{C}$ \\
II & 21,1 & 25,0 & 20,8 & 22,2 & $22,2 \mathrm{~B}$ \\
III & 21,8 & 22,6 & 23,9 & 24,3 & $23,1 \mathrm{~A}$ \\
Media & $18,4 \mathrm{c}$ & $20,5 \mathrm{a}$ & $19,2 \mathrm{bc}$ & $19,9 \mathrm{ab}$ & 19,5 \\
\hline
\end{tabular}

${ }^{1}$ Los valores seguidos por la misma letra minúscula en la fila y mayúsculas en la columna no son significativamente diferentes por el test de Bonferroni al nivel de $5 \%$ de probabilidad.

CUADRO 4. Valores medios del contenido de carbono total $(\mathrm{g} / \mathrm{kg})$ en los sistemas de cultivo Cebada/Cebada (Sistema I), Veza-Avena/Girasol (Sistema II) y Prado/Veza-Avena (Sistema III) en distintas épocas de muestreo. Madrid, 1996'.

\begin{tabular}{cccccc}
\hline Sistemas & \multicolumn{5}{c}{ Carbono total $(\mathrm{g} / \mathrm{kg})$} \\
\cline { 2 - 6 } & Feb.-93 & Sep.-93 & Feb.-94 & Sep.-94 & Media \\
\hline I & 44,0 & 47,2 & 66,0 & 69,6 & $56,7 \mathrm{~B}$ \\
II & 57,3 & 63,0 & 73,5 & 73,8 & $66,9 \mathrm{~A}$ \\
III & 62,2 & 62,9 & 79,1 & 57,3 & $65,4 \mathrm{~A}$ \\
Media & $54,5 \mathrm{~d}$ & $57,7 \mathrm{c}$ & $72,8 \mathrm{a}$ & $66,9 \mathrm{~b}$ & 63,0
\end{tabular}

${ }^{1}$ Los valores seguidos por la misma letra minúscula en la fila y mayúsculas en la columna no son significativamente diferentes por el test de Bonferroni al nivel de $5 \%$ de probabilidad. 
(Carbono oxidable entre carbono del extracto húmico total) que se recoge en el Cuadro 10. Cuanto menor sea este, mayor es la cantidad de carbono de materia orgánica que está en forma humificada. El sistema III es el que presenta el mayor grado de humificación, seguido de los sistemas I y II (Guerrero, 1987).

En cuanto al grado de evolución de la materia orgánica humificada $\left(\mathrm{C}_{\mathrm{EHT}} / \mathrm{C}_{\mathrm{AH}}\right)$ estará más evolucionada, más polimerizada, cuanto menor sea este valor. Encontramos que en el sistema II existe una mayor polimerización con diferencia significativa en relación a los sistemas I y III (Cuadro 11). Entre los sistemas I y III no se observa diferencias con relación al grado de polimerización.

La relación del carbono del extracto húmico total $\left(\mathrm{C}_{\mathrm{EHT}}\right)$ frente al carbono oxidable es lo que se denomina "tasa de extracción", cuyos valores para los tres sistemas de cultivo y épocas de mustreo se recogen en el Cuadro 12. Se observan diferencias entre los sistemas de cultivo, con una mayor "tasa de extracción" en el sistema III. Los menores valores se aprecian en el sistema II, pero todos ellos estan cerca de los $30 \%$, que es la tasa de extracción normal en el suelo (Guerrero et al., 1979; Díaz-Marcote, 1994).

Entre sistemas las diferencias son significativas y concordantes con los contenidos en materia orgánica del suelo. Los valores crecen en el sentido I, II y III, concordante con los contenidos de materia orgánica del suelo y el carbono del extracto húmico total.

En el Cuadro 13 se muestran los coeficientes de correlación entre las propiedades químicas estudiadas. Se verifica correlaciones significativas y

CUADRO 5. Valores medios del contenido de carbono orgánico en el extracto húmico total en los sistemas de cultivo Cebada/Cebada (Sistema I), Veza-Avena/Girasol (Sistema II) y Prado/Veza-Avena (Sistema III) en distintas épocas de muestreo. Madrid, 1996 ${ }^{1}$.

\begin{tabular}{cccccc}
\hline Sistemas & \multicolumn{5}{c}{ Carbono orgánico en el extracto húmico total $(\mathrm{g} / \mathrm{kg})$} \\
\cline { 2 - 6 } & Feb.-93 & Sep.-93 & Feb.-94 & Sep.-94 & Media \\
\hline I & 2,7 & 2,2 & 2,2 & 2,4 & $2,4 \mathrm{C}$ \\
II & 3,0 & 3,6 & 4,0 & 4,7 & $3,8 \mathrm{~B}$ \\
III & 4,5 & 4,4 & 4,2 & 4,7 & $4,4 \mathrm{~A}$ \\
Media & $3,4 \mathrm{~b}$ & $3,4 \mathrm{~b}$ & $3,5 \mathrm{~b}$ & $3,9 \mathrm{a}$ & 3,5 \\
\hline
\end{tabular}

${ }^{1}$ Los valores seguidos por la misma letra minúscula en la fila y mayúsculas en la columna no son significativamente diferentes por el test de Bonferroni al nivel de $5 \%$ de probabilidad.

CUADRO 6. Valores medios del contenido de carbono orgánico en los ácidos húmicos (g/kg) en los sistemas de cultivo Cebada/Cebada (Sistema I), Veza-Avena/Girasol (Sistema II) y Prado/Veza-Avena (Sistema III) en distintas épocas de muestreo. Madrid, 1996'.

\begin{tabular}{cccccc}
\hline \multirow{2}{*}{ Sistemas } & \multicolumn{5}{c}{ Carbono orgánico en los ácidos húmicos $(\mathrm{g} / \mathrm{kg})$} \\
\cline { 2 - 5 } & Feb.-93 & Sep.-93 & Feb.-94 & Sep.-94 & Media \\
\hline I & 1,5 & 1,6 & 1,2 & 1,2 & $1,4 \mathrm{~B}$ \\
II & 1,9 & 2,5 & 2,8 & 3,8 & $2,7 \mathrm{~A}$ \\
III & 2,4 & 2,5 & 2,5 & 3,4 & $2,7 \mathrm{~A}$ \\
Media & $1,9 \mathrm{c}$ & $2,2 \mathrm{~b}$ & $2,1 \mathrm{bc}$ & $2,8 \mathrm{a}$ & 2,3 \\
\hline
\end{tabular}

${ }^{1}$ Los valores seguidos por la misma letra minúscula en la fila y mayúsculas en la columna no son significativamente diferentes por el test de Bonferroni al nivel de $5 \%$ de probabilidad. 
CUADRO 7. Valores medios del contenido de nitrógeno $(\mathrm{g} / \mathrm{kg})$ en los sistemas de cultivo Cebada/Cebada (Sistema I), Veza-Avena/Girasol (Sistema II) y Prado/Veza-Avena (Sistema III) en distintas épocas de muestreo. Madrid, 1996 ${ }^{1}$.

\begin{tabular}{cccccc}
\hline Sistemas & \multicolumn{5}{c}{ Nitrógeno $(\mathrm{g} / \mathrm{kg})$} \\
\cline { 2 - 6 } & Feb.-93 & Sep.-93 & Feb.-94 & Sep.-94 & Media \\
\hline I & 0,9 & 0,7 & 0,6 & 0,9 & $0,8 \mathrm{~B}$ \\
II & 1,4 & 1,1 & 1,0 & 1,3 & $1,2 \mathrm{~A}$ \\
III & 1,3 & 1,2 & 1,1 & 1,4 & $1,3 \mathrm{~A}$ \\
Media & $1,2 \mathrm{a}$ & $1,0 \mathrm{~b}$ & $0,9 \mathrm{~b}$ & $1,2 \mathrm{a}$ & 1,1 \\
\hline
\end{tabular}

${ }^{1}$ Los valores seguidos por la misma letra minúscula en la fila y mayúsculas en la columna no son significativamente diferentes por el test de Bonferroni al nivel de $5 \%$ de probabilidad.

CUADRO 8. Valores medios de la capacidad de intercambio catiónico $\left(\mathrm{mmol}_{\mathrm{c}} / \mathrm{kg}\right)$ en los sistemas de cultivo Cebada/Cebada (Sistema I), Veza-Avena/Girasol (Sistema II) y Prado/Veza-Avena (Sistema III) en distintas épocas de muestreo. Madrid, 1996¹.

\begin{tabular}{cccccc}
\hline Sistemas & \multicolumn{5}{c}{ Capacidad de intercambio catiónico $\left(\mathrm{mmol}_{\mathrm{c}} \mathrm{kg}\right)$} \\
\cline { 2 - 5 } & Feb.-93 & Sep.-93 & Feb.-94 & Sep.-94 & Media \\
\hline I & 79,6 & 68,4 & 75,6 & 77,7 & $75,3 \mathrm{C}$ \\
II & 118,9 & 85,5 & 117,0 & 126,4 & $111,9 \mathrm{~B}$ \\
III & 141,9 & 120,0 & 128,5 & 132,3 & $130,7 \mathrm{~A}$ \\
Media & $113,4 \mathrm{a}$ & $91,3 \mathrm{~b}$ & $107,0 \mathrm{a}$ & $112,1 \mathrm{a}$ & 106,0 \\
\hline
\end{tabular}

${ }^{1}$ Los valores seguidos por la misma letra minúscula en la fila y mayúsculas en la columna no son significativamente diferentes por el test de Bonferroni al nivel de $5 \%$ de probabilidad.

CUADRO 9. Valores medios de la relación carbono/nitrógeno en los sistemas de cultivo Cebada/Cebada (Sistema I), Veza-Avena/Girasol (Sistema II) y Prado/Veza-Avena (Sistema III) en distintas épocas de muestreo. Madrid, 1996'.

\begin{tabular}{cccccc}
\hline Sistemas & \multicolumn{5}{c}{ Relación carbono/nitrógeno } \\
\cline { 2 - 6 } & Feb.-93 & Sep.-93 & Feb.-94 & Sep.-94 & Media \\
\hline I & 8,58 & 12,13 & 13,13 & 8,31 & $10,54 \mathrm{~A}$ \\
II & 8,86 & 13,67 & 12,67 & 9,95 & $11,29 \mathrm{~A}$ \\
III & 10,05 & 11,54 & 12,59 & 10,11 & $11,07 \mathrm{~A}$ \\
Media & $9,16 \mathrm{~b}$ & $12,44 \mathrm{a}$ & $12,80 \mathrm{a}$ & $9,46 \mathrm{~b}$ & 10,97 \\
\hline
\end{tabular}

${ }^{1}$ Los valores seguidos por la misma letra minúscula en la fila y mayúsculas en la columna no son significativamente diferentes por el test de Bonferroni al nivel de $5 \%$ de probabilidad. 
CUADRO 10. Valores medios del grado de humificación $\left(\mathrm{C}_{\mathrm{ox}} / \mathrm{C}_{\mathrm{EHT}}\right)$ en los sistemas de cultivo Cebada/Cebada (Sistema I), Veza-Avena/Girasol (Sistema II) y Prado/Veza-Avena (Sistema III) en distintas épocas de muestreo. Madrid, 1996.

\begin{tabular}{cccccc}
\hline Sistemas & \multicolumn{5}{c}{ Grado de humificación $\left(\mathrm{C}_{\mathrm{ox}} / \mathrm{C}_{\mathrm{EHT}}\right)$} \\
\cline { 2 - 5 } & Feb.-93 & Sep.-93 & Feb.-94 & Sep.-94 & Media \\
\hline I & 2,73 & 4,01 & 3,47 & 3,17 & $3,34 \mathrm{~A}$ \\
II & 4,16 & 4,16 & 3,07 & 2,76 & $3,54 \mathrm{~A}$ \\
III & 2,85 & 3,10 & 3,33 & 3,00 & $3,07 \mathrm{~B}$ \\
Media & $3,25 \mathrm{bc}$ & $3,75 \mathrm{a}$ & $3,29 \mathrm{~b}$ & $2,98 \mathrm{c}$ & 3,32 \\
\hline
\end{tabular}

${ }^{1}$ Los valores seguidos por la misma letra minúscula en la fila y mayúsculas en la columna no son significativamente diferentes por el test de Bonferroni al nivel de $5 \%$ de probabilidad.

CUADRO 11. Valores medios del grado de evolución de la materia orgánica humificada $\left(\mathrm{C}_{\mathrm{EHT}} / \mathrm{C}_{\mathrm{AH}}\right)$ en los sistemas de cultivo Cebada/Cebada (Sistema I), Veza-Avena/Girasol (Sistema II) y Prado/Veza-Avena (Sistema III) en distintas épocas de muestreo. Madrid, 1996 1 .

\begin{tabular}{cccccc}
\hline Sistemas & \multicolumn{5}{c}{ Grado de evolución de la materia orgánica humificada $\left(\mathrm{C}_{\mathrm{EHT}} / \mathrm{C}_{\mathrm{AH}}\right)$} \\
\cline { 2 - 5 } & Feb.-93 & Sep.-93 & Feb.-94 & Sep.-94 & Media \\
\hline I & 1,86 & 1,47 & 2,12 & 2,09 & $1,89 \mathrm{~A}$ \\
II & 1,64 & 1,48 & 1,47 & 1,24 & $1,47 \mathrm{~B}$ \\
III & 1,89 & 1,74 & 1,71 & 1,41 & $1,69 \mathrm{~A}$ \\
Media & $1,80 \mathrm{a}$ & $1,56 \mathrm{a}$ & $1,77 \mathrm{a}$ & $1,58 \mathrm{a}$ & 1,68 \\
\hline
\end{tabular}

${ }^{1}$ Los valores seguidos por la misma letra minúscula en la fila y mayúsculas en la columna no son significativamente diferentes por el test de Bonferroni al nivel de $5 \%$ de probabilidad.

CUADRO 12. Valores medios para la relación $\mathrm{C}_{\mathrm{EHT}} / \mathrm{C}_{\mathrm{ox}}$ en los sistemas de cultivo Cebada/Cebada (Sistema I), Veza-Avena/Girasol (Sistema II) y Prado/Veza-Avena (Sistema III) en distintas épocas de muestreo. Madrid, 1996.

\begin{tabular}{cccccc}
\hline Sistemas & \multicolumn{5}{c}{ Relación $\left(\mathrm{C}_{\mathrm{EHT}} / \mathrm{C}_{\mathrm{ox}}\right)$} \\
\cline { 2 - 6 } & Feb.-93 & Sep.-93 & Feb.-94 & Sep.-94 & Media \\
\hline I & 0,38 & 0,26 & 0,29 & 0,32 & $0,31 \mathrm{AB}$ \\
II & 0,24 & 0,24 & 0,33 & 0,37 & $0,29 \mathrm{~B}$ \\
III & 0,36 & 0,33 & 0,31 & 0,34 & $0,33 \mathrm{~A}$ \\
Media & $0,33 \mathrm{ab}$ & $0,28 \mathrm{c}$ & $0,31 \mathrm{~b}$ & $0,34 \mathrm{a}$ & 0,31 \\
\hline
\end{tabular}

${ }^{1}$ Los valores seguidos por la misma letra minúscula en la fila y mayúsculas en la columna no son significativamente diferentes por el test de Bonferroni al nivel de $5 \%$ de probabilidad. 
CUADRO 13. Matriz de correlaciones correspondientes a las variables: CE (conductividad eléctrica en $\mu \mathrm{S} / \mathrm{cm}$ ), pH, MO (materia orgánica en $\mathrm{g} / \mathrm{kg}$ ), CT (carbono total a $540^{\circ} \mathrm{C}$ en $\mathrm{g} / \mathrm{kg}$ ), EHT (carbono en el extracto húmico total en $\mathrm{g} / \mathrm{kg}$ ), AH (carbono en los ácidos húmicos en $\mathrm{g} / \mathrm{kg}$ ), AF (carbono en los ácidos fúlvicos en $\mathrm{g} / \mathrm{kg}$ ), $\mathrm{N}$ (nitrógeno en $\mathrm{g} / \mathrm{kg}$ ), C/N (relación entre carbono y nitrógeno), CIC (capacidad de intercambio catiónico en mmol $/ \mathrm{kg}$ ), $\mathrm{C}_{\text {ox }}$ EHT (relación entre carbono oxidable y EHT), EHT/AH (relación entre EHT y AH) y EHT/C ${ }_{\text {ox }}$ (relación entre EHT y carbono oxidable). Madrid. 1996.

\begin{tabular}{|c|c|c|c|c|c|c|c|c|c|c|c|c|c|}
\hline Atributo & $\mathrm{CE}$ & $\mathrm{pH}$ & MO & CT & EHT & $\mathrm{AH}$ & $\mathrm{AF}$ & $\mathrm{N}$ & $\mathrm{C} / \mathrm{N}$ & CIC & $\mathrm{C}_{\mathrm{ox}} / \mathrm{EHT}$ & EHT/AH & $\mathrm{EHT} / \mathrm{C}_{\mathrm{ox}}$ \\
\hline $\mathrm{EHT} / \mathrm{C}_{\mathrm{ox}}$ & $0,237^{\mathrm{NS}}$ & $0,101^{\mathrm{NS}}$ & $-0,124^{\mathrm{NS}}$ & $-0,022^{\mathrm{NS}}$ & $0,471 * * *$ & $0,284^{*}$ & $0,444 * * *$ & $0,002^{\mathrm{NS}}$ & $-0,220^{\mathrm{NS}}$ & $0,281^{*}$ & $-0,951 * *$ & $1,000 * * *$ & 1,000 \\
\hline EHT/AH & $-0,083^{\mathrm{NS}}$ & $0,133^{\mathrm{NS}}$ & $-0,371 * * *$ & $-0,004^{\mathrm{NS}}$ & $-0,238^{\mathrm{NS}}$ & $-0,654 * * *$ & $0,573^{* * *}$ & $-0,775^{* * *}$ & $-0,322^{*}$ & $-0,235^{\mathrm{NS}}$ & $-0,209^{\mathrm{NS}}$ & 1,000 & \\
\hline $\mathrm{C}_{\mathrm{ox}} / \mathrm{EHT}$ & $-0,261 *$ & $-0,043^{\mathrm{NS}}$ & $0,104^{\mathrm{NS}}$ & $-0,074^{\mathrm{NS}}$ & $-0,475^{* * *}$ & $-0,290^{*}$ & $-0,447 * * *$ & $-0,044^{\mathrm{NS}}$ & $0,245^{\mathrm{NS}}$ & $-0,308^{*}$ & 1,000 & & \\
\hline CIC & $0,588^{* * * *}$ & $-0,490 * * *$ & $0,730^{* * *}$ & $0,385^{* *}$ & $0,830^{* * * *}$ & $0,694 * * *$ & $0,482 * * *$ & $0,727 * * *$ & $-0,041^{\mathrm{NS}}$ & 1,000 & & & \\
\hline $\mathrm{C} / \mathrm{N}$ & $-0,341 * *$ & $0,125^{\mathrm{NS}}$ & $0,269^{*}$ & $0,237^{\mathrm{NS}}$ & $0,102^{\mathrm{NS}}$ & $0,086^{\mathrm{NS}}$ & $0,044^{\mathrm{NS}}$ & $-0,402 * *$ & 1,000 & & & & \\
\hline $\mathrm{N}$ & $0,632^{* * * *}$ & $-0,452^{* * *}$ & $0,759^{* * *}$ & $0,247^{\mathrm{NS}}$ & $0,675^{* * *}$ & $0,626^{* * *}$ & $0,296^{*}$ & 1,000 & & & & & \\
\hline $\mathrm{AF}$ & $0,352^{* *}$ & $-0,119^{\mathrm{NS}}$ & $0,346^{* *}$ & $0,225^{\mathrm{NS}}$ & $0,558 * * *$ & $0,031^{\mathrm{NS}}$ & 1,000 & & & & & & \\
\hline $\mathrm{AH}$ & $0,440 * * *$ & $-0,242^{\mathrm{NS}}$ & $0,739^{* * *}$ & $0,342 * *$ & $0,846^{* * * *}$ & 1,000 & & & & & & & \\
\hline EHT & $0,549 * * *$ & $-0,267 *$ & $0,801 * * *$ & $0,395 * *$ & 1,000 & & & & & & & & \\
\hline $\mathrm{CT}$ & $0,056^{\mathrm{NS}}$ & $-0,241^{\mathrm{NS}}$ & $0,437^{* * *}$ & * 1,000 & & & & & & & & & \\
\hline $\mathrm{MO}$ & $0,426^{* * * *}$ & $-0,382 * *$ & 1,000 & & & & & & & & & & \\
\hline $\mathrm{pH}$ & $-0,335 * *$ & 1,000 & & & & & & & & & & & \\
\hline $\mathrm{CE}$ & 1,000 & & & & & & & & & & & & \\
\hline
\end{tabular}


positivas, al nivel de $0,1 \%$, entre el contenido de materia orgánica y conductividad eléctrica, capacidad de intercambio catiónico, carbono del extracto húmico total, carbono de los ácidos húmicos, nitrógeno y carbono total. La correlación entre materia orgánica y los valores de $\mathrm{pH}$ es negativa y significativa al nivel de $1 \%$, indicativo del efecto acidificante de la fracción orgánica del suelo.

Las correlaciones positivas más fuertes se observan entre: capacidad de intercambio catiónico y carbono del extracto húmico total; materia orgánica y carbono del extracto húmico total; carbono de los ácidos húmicos y carbono del extracto húmico total; relación $\mathrm{EHT} / \mathrm{C}_{\mathrm{ox}}$ y el índice que expresa el grado de humificación de la materia orgánica $\left(\mathrm{C}_{\mathrm{EHT}} / \mathrm{C}_{\mathrm{AH}}\right)$. Todos con valores superiores a 0,8 . Queda establecida la interdependencia de la materia orgánica del suelo, en cantidad y en calidad, con una serie de parámetros químicos de los suelos.

\section{CONCLUSIONES}

1. La materia orgánica del sistema Prado/Veza-Avena (Sistema III) presenta el menor coeficiente de variación con respecto a los sistemas Cebada/Cebada (Sistema I) y Veza-Avena/Girasol (Sistema II).

2. El contenido de materia orgánica, a lo largo del tiempo, de los sistemas Prado/Veza-Avena (Sistema III) y Veza-Avena/Girasol (Sistema II) representa un $42,86 \%$ y un $40,54 \%$ más que la sucessión Cebada/Cebada (Sistema I), que presenta los menores valores de capacidad de intercambio catiónico, contenido en carbono total y para el extracto húmico total.

3. La materia orgánica del sistema Prado/Veza-Avena (Sistema III) es la de mayor grado de humificación y la del sistema II la de mayor grado de polimerización.

4. El menor contenido de nitrógeno orgánico es el del sistema I (Cebada/Cebada), no encontrándose diferencias entre las relaciones $\mathrm{C} / \mathrm{N}$.

\section{REFERENCIAS}

ALMENDROS, G.; IBAÑEZ, J.J.; POLO, A. Contribución al estudio del subsistema edáfico en los ecosistemas climácticos del Sistema Central (España). II. Caracterización fisicoquímica del humus en los ecosistemas del Puerto de la Quesera (Macizo de Ayllón). Revue d'Ecologie et de Biologie du Sol, v.19, n.2, p.151-162, 1982.

ANUARIO DE ESTADÍSTICA AGRARIA. Madrid: Ministerio de Agricultura Pesca y Alimentación, 1990. p.23-27.

BERNAL, M.P.; ROIG, A.; LAX, A.; NAVARRO, A.F. Effects of the applications of pig slurry on some physico-chemical properties of calcareous soils. Bioresource Technology, v.42, p.233-239, 1992.

CARVALHO, O.S. Variabilidad espacial y temporal de la materia orgánica en un Typic Xerofluvent sometido a distintas sucessiones de cultivo. Madrid: Universidad Politécnica de Madrid, Escuela Técnica Superior de Ingenieros Agrónomos, 1996. 140p. Tesis Doctoral.

COSTA, F.; GARCÍA, C.; HERNÁNDEZ, T.; POLO, A. Residuos orgánicos urbanos: manejo y utilización. Murcia: Consejo Superior de Investigaciones Científicas, 1991. 181p.

DABIN, B. Étude d'une méthode d'extraction de la matiere humique du sol. Science du Sol, v.1, p.47-63, 1971.

DAVIES, B.; EAGLE, D.; FINNEY, B. Manejo del suelo. Buenos Aires: Ateneo, 1987. 228p.

DÍAZ-MARCOTE, I. Aprovechamiento del compost de los residuos sólidos urbanos: estudio de su capacidad fertilizante y del efecto sobre las propiedades físicas, químicas y biológicas del suelo. Madrid: Universidad Politécnica de Madrid, Escuela Técnica Superior de Ingenieros Agrónomos, 1994. 281p. Tesis Doctoral.

DUCHAUFOUR, P. Manual de edafología. Barcelona: Masson, 1987. 214p.

ESPANHA. Ministerio de Agricultura Pesca y ALIMENTACIÓN. Métodos oficiales de análisis. Madrid, 1986. 532p.

FAO. (Roma, Itália). Manejo del suelo: producción y uso del composte en ambientes tropicales y subtropicales. Roma, 1991. 178p. (FAO. Boletín de Suelos, 56).

FUENTES, J.L.Y. Técnicas de riego. Madrid: IRYDA, 1992. 351p.

GONZÁLEZ, M.I.M.; GALLARDO, J.F.; EGIDO, J.A. Evolución de la materia orgánica de un suelo climax y de un suelo de repoblación de la vertiente norte de la 
Sierra de Gata (Salamanca). Anales de Edafología y Agrobiología, v.36, n.11/12, p.1167-1186, 1977.

GUERRERO, F. Estudio de las propiedades físicas y químicas de algunas turbas españolas y su posible aprovechamiento agrícola. Madrid: Universidad Autónoma de Madrid, Facultad de Ciencias, 1987. 222p. Tesis Doctoral.

GUERRERO, M.C.; POLO, A.; VELASCO, F. Estudio de tres humus representativos del centro de España y su población microbiana. Agrochimica, v.23, n.5/6, p.401-411, 1979.

HERNANDO, S. Aprovechamiento de residuos sólidos urbanos como fuente de materia orgánica y sus efectos sobre las propiedades físicas y químicas del suelo. Madrid: Universidad Autónoma de Madrid, 1987. 230p. Tesis Doctoral.

JORGE, J.A. Física e manejo dos solos tropicais. Campinas, SP: Instituto Campineiro de Ensino Agrícola, 1986. 328p.

LOBO, M. del C. Biodegradación y humificación del sarmento de vid. Caracterización agrobiológica de los compost obtenidos. Madrid: Universidad Autónoma de Madrid, Facultad de Ciencias, 1985. 203p. Tesis Doctoral.

LÓPEZ-FANDO, C. Degradación de un xeralf sometido a distintas técnicas agrícolas. Madrid: Universidad Politécnica de Madrid, Escuela Técnica Superior de Ingenieros Agrónomos, 1991. 223p. Tesis Doctoral.

MELO, W.J.; MARQUES, M.O.; SANTIAGO, G.; CHELI, R.A.; LEITE, S.A.S. Efeito de doses crescentes de lodo de esgoto sobre frações da matéria orgânica e CTC de un latossolo cultivado com cana-de-açúcar. Revista Brasileira de Ciência do Solo, v.18, p.449-455, 1994.

SAS INSTITUTE. SAS user's guide: statistics. Version 5. ed. Cary, 1985. 956p.

URBANO, P.; MORO, R. Sistemas agrícolas con rotaciones $y$ alternativas de cultivo. Madrid: Mundi-Prensa, 1992. 134p.

WILD, A. Condiciones del suelo y desarrollo de las plantas según Russel. Madrid: Mundi-Prensa, 1992. 1045 p. 